\title{
Three Megapixel Ultrasonic Microscope Imaging
}

\section{Hyvönen, Jere}

IEEE

2019

Hyvönen , J , Meriläinen , A , Salmi , A , Hupa , L , Lindfors , N \& Haeggström , E 2019 , Three Megapixel Ultrasonic Microscope Imaging . in 2019 IEEE International Ultrasonics Symposium (IUS) . IEEE International Ultrasonics Symposium , IEEE , NEW YORK, pp. 1886-1889 , IEEE International Ultrasonics Symposium , Glasgow , United Kingdom , 06/10/2019 . https://doi.org/10.1109/ULTSYM.2019.8925785

http://hdl.handle.net/10138/326123

https://doi.org/10.1109/ULTSYM.2019.8925785

acceptedVersion

Downloaded from Helda, University of Helsinki institutional repository.

This is an electronic reprint of the original article.

This reprint may differ from the original in pagination and typographic detail.

Please cite the original version. 


\section{Three Megapixel Ultrasonic Microscope Imaging}

\author{
Jere Hyvönen \\ Electronics Research Laboratory, \\ Department of Physics \\ The University of Helsinki \\ Helsinki, Finland \\ jere.hyvonen@helsinki.fi
}

\author{
Antti Meriläinen \\ Electronics Research Laboratory, \\ Department of Physics \\ The University of Helsinki \\ Helsinki, Finland \\ antti.merilainen@helsinki.fi
}

Nina Lindfors

Helsinki University Hospital

The University of Helsinki

Helsinki, Finland

\author{
Ari Salmi \\ Electronics Research Laboratory, \\ Department of Physics \\ The University of Helsinki \\ Helsinki, Finland \\ ari.salmi@helsinki.fi \\ Edward Hæggström \\ Electronics Research Laboratory, \\ Department of Physics \\ The University of Helsinki \\ Helsinki, Finland
}

\author{
Leena Hupa \\ Process Chemistry Centre, \\ Åbo Akademi University, \\ Turku, Finland
}

\begin{abstract}
Acoustic microscopy is a modality that utilizes mechanical properties as the imaging contrast. Imaging large areas is desirable for biological samples which often have features that span large areas $\left(>1 \mathrm{~mm}^{2}\right)$. Here we use our custom-made coded excitation scanning acoustic microscope (CESAM) for large area scanning: we visualized two bioactive glass ( $\mathrm{S53P4}$ ) bone substitutes in a rabbit femur, the new bone formation in the bioactive glass, as well as the surrounding bone after implantation. In this study we used $375 \mathrm{MHz}$ central frequency transducer and a 300 to $500 \mathrm{MHz}$ linear chirp with a gaussian envelope. The sample was scanned in 10 different focus layers, each $10 \mu \mathrm{m}$ apart from each other. These 10 layers were aligned using 2D-cross correlation. In the final image. The reconstruction was done by picking the A-line in focus for each pixel. We discuss the method for large area scanning with focus layer alignment. We show that it reduces the sample flatness requirements. Absolute acoustic impedance values were calculated from the measured data via acoustic impedance calibration [6]. We demonstrated that our device is capable of $250+\mathrm{mm}^{2}$ area imaging with $10 \mu \mathrm{m} \mathrm{XY-}$ stepping, with sample surface height variation of over $100 \mu \mathrm{m}$.
\end{abstract}

Keywords-Acoustic Microscopy, multi-layer, bone, imaging, signal-processing

\section{INTRODUCTION}

Biological samples feature uneven surfaces. This is a problem when using traditional acoustic lenses to produce a focusing beam with high imaging resolution. The working distance gets smaller as the resolution gets higher. Coded excitation scanning acoustic microscopy (CESAM) enables fast measurement times and high signal-to-noise ratios (SNR) due to usage of long codes and cross-correlation which reduces the need of averaging[1]. Bioactive glasses (BAG) are well known bone substitutes possessing favourable qualities for bone formation. Due to the surface reactions starting after implantation they are known to chemically bond to bone. After implantation a rapid exchange of $\mathrm{Na}^{+}$and $\mathrm{K}^{+}$in the glass with $\mathrm{H}^{+}$and $\mathrm{H}_{3} \mathrm{O}^{+}$from the extracellular solution takes place leading to formation of silanol $(\mathrm{SiOH})$ groups at the glass surface. The silanol groups then repolymerize into a silica-rich layer, followed by migration of $\mathrm{Ca}^{2+}$ and $\mathrm{PO}_{4}{ }^{3-}$ groups to crystallise into a $\mathrm{CaO}-\mathrm{P}_{2} \mathrm{O}_{5}$ hydroxyapatite (HAP) layer on top of the silicarich layer. Cell interactions with the HAP layer then initiate the bone forming pathway. Moreover, BAG-S53P4 can stimulate bone formation, angiogenesis and it also possesses antibacterial properties. [2-4]

\section{METHODS}

\section{A. Custom-built Scanning Acoustic Microscope at 0.1 to 1 $\mathrm{GHz}$}

We used a custom-built Coded Excitation Scanning Acoustic Microscope (CESAM) whose first version was introduced in [5]. The device hardware and software ensemble are illustrated in Figure 1. The hardware control and data acquisition and analysis software were written in MATLAB 9.2 (Mathworks). The signal transmission and data acquisition were done by a signal generator (Spectrum M4i.6631-x8) and an oscilloscope (Spectrum M4i.2233-x8) respectively. The transmit signals first went through a frequency doubler (Mini Circuits ZX90-213-S) followed with an amplifier (Mini Circuits ZHL-42W) and a custom-built T/R switch. A transducer transmit and received the ultrasound signals. The $T / R$ switch guided the received signals to another channel where they were amplified with a pre-amplifier (Mini Circuits ZFL-1000LN+) before recording. In order to perform $\mathrm{C}$-scans in multiple focal layers the sample was moved under a transducer in $\mathrm{XYZ}$ - dimension with a $2 \mathrm{D}$ - linear stage (Thorlabs MLS203-1 and BBD202) for XY and a 1D-linear stage (Thorlabs MTS 25/M-Z8 and KDC101) for Z which were controlled by an Arduino due. The Arduino tracked the motor encoder pulses to trigger a pulse echo (PE) measurement in a correct position. The whole device was built on an optical table (Thorlabs SDA7590 frame, B7590L plate) to minimize any vibrations that could affect the measurement. Sample and transducer are aligned with the scanning axis using goniometers (Thorlabs GN2/M). 


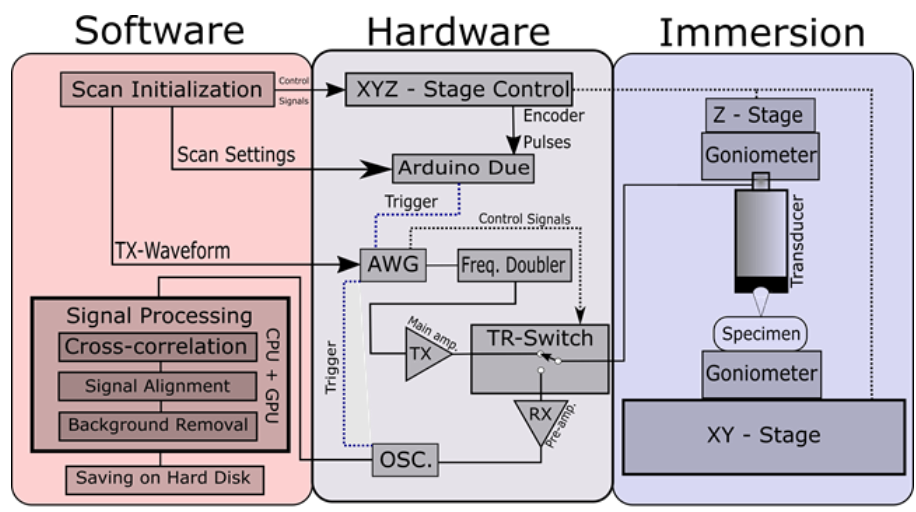

Fig. 1. Schematic of the Coded Excitation Scanning Acoustic Microscope (CESAM) describing the working principle of the device.

The CESAM employs coded excitation to increase SNR [1]. Here a $1 \mu$ s long $300-500 \mathrm{MHz}$ linear chirp with a gaussian envelope was transmit to a $400 \mathrm{MHz}$ single element transducer with a spherical sapphire lens structure (Kibero). The device performs on-the-fly PE measurements at a rate of approximately 1000 a-lines/second allowing to image 3 megapixel C-scans in 50 minutes.

\section{B. $\quad$ Samples and imaging methods}

We had two different rabbit femur samples. One embedded in plastic and one fresh sample stored in ethanol. Both samples were from individuals that had gone through an insertion of a glass implant operation.

The BAG-S53P4, (53 wt- $\% \mathrm{SiO}_{2}, 23$ wt- $\% \mathrm{NaO}, 20$ wt- $\%$ $\mathrm{CaO}$ and $4 \%$ wt- $\mathrm{P}_{2} \mathrm{O}_{5}$ ) was made from mixtures of Belgian sand for $\mathrm{SiO}_{2}$, and analytical grades of $\mathrm{Na}_{2} \mathrm{CO}_{3}, \mathrm{CaCO}_{3}$ and $\mathrm{CaHPO}_{4} \cdot 2 \mathrm{H}_{2} \mathrm{O}$. The mixture was melted in a platinum cruicible for $3 \mathrm{~h}$ at $1360^{\circ} \mathrm{C}$, which after the glass was preheated in graphite mold, crushed and remelted for homogenization. The glass was re-crushed and sieved to a particle size of $300-500 \mu \mathrm{m}$. Cylindrical rods of $15 \times 5 \mathrm{~mm}$ and $10 \times 10 \mathrm{~mm}$ size were obtained by sintering the granules in graphite molds at $720^{\circ} \mathrm{C}$ for $90 \mathrm{~min}$ and $630^{\circ} \mathrm{C}$ for $60 \mathrm{~min}$ respectively.

Two rabbits (NZW, Harlan laboratories) were used. The procedures were performed under general anesthesia using subcutaneous medetomidine- and ketamine hydrochloride. In one rabbit a bone defect was performed by drilling a 6-mm horizontal hole from the lateral cortical side into the metaphyseal region of the femur. A rod of $15 \times 5 \mathrm{~mm}$ size was implanted in the drilled hole. In another rabbit a diaphyseal defect was obtained by removing a $10 \mathrm{~mm}$ piece of the diaphyse of the femur, which after a $10 \times 10 \mathrm{~mm}$ rod was implanted in the defect. The femur and the bone substitute were stabilized by a plate and six locking screws. Postoperatively buprenorphine and procain penicillin was given for three days. The animals were euthanized with an overdose of pentobarbital at 8 weeks. Samples of the implants were then taken for further analysis.

The study was approved by the Animal Experimental Board of Finland (ESAVI/440/04.10.07/2014) and (ESAVI/642304.10.07/2017). The principles of laboratory animal care of Helsinki University were followed.
The samples were attached to a plastic container with cyanoacrylate in order to prevent unwanted motion of the sample. The measurements were done in PBS (phosphate buffered saline) to minimize the osmosis effect on the sample. First the sample and transducer were aligned with B-scans utilizing the goniometers to which they are attached to. In optimal case the focal plane is parallel with the translation stage axes. A stack of C-scans was measured one focal plane

at a time, starting from negative defocus. Then the transducer was moved further away with 10 or $15 \mu \mathrm{m}$ steps at the end of each C-scan. This way we constructed a stack of A-lines where each point of the sample is measured at focus. The focal echo amplitude was calibrated using samples with different acoustic impedance values thus different acoustic reflection coefficients [6].

\section{Data Analysis}

The data was first pre-processed with 3 steps:

1. Cross-correlation of the measured echoes and the transmit waveform

2. Signal alignment with the 1 . lens echo as a reference point

3. Background removal from a measurement done without a reflector.

Then the amplitude and TOF values were extracted by fitting 2nd order polynomials to the lens and focal echoes. Given a stack of amplitudes and corresponding TOFs the amplitude images are formed by picking pixels that are in focus. The loss of amplitude due to defocus is compensated with the known form of the $\mathrm{V}(\mathrm{z})$-curve that is measured from an ideal reflector. This compensated amplitude used to compute the corresponding calibrated acoustic reflection coefficient.

\section{RESULTS AND DISCUSSION}

\section{A. Example 1: Epoxy cast BAG-S53P4 femur sample}

In Fig. 2 the bone structure in the sample resembles the trabecular or spongy bone with open spaces, which is characteristic for the metaphyseal part of long bones. BAG granules are clearly visible in the implant. A reaction layer is also visible on the BAG granules, which is in accordance with previous observations made e.g. using scanning electron microscopy or histology [7]. Interestingly, the untouched bone formation (to the left) visualizes as an anatomical tissue with a clearly organized structure. 


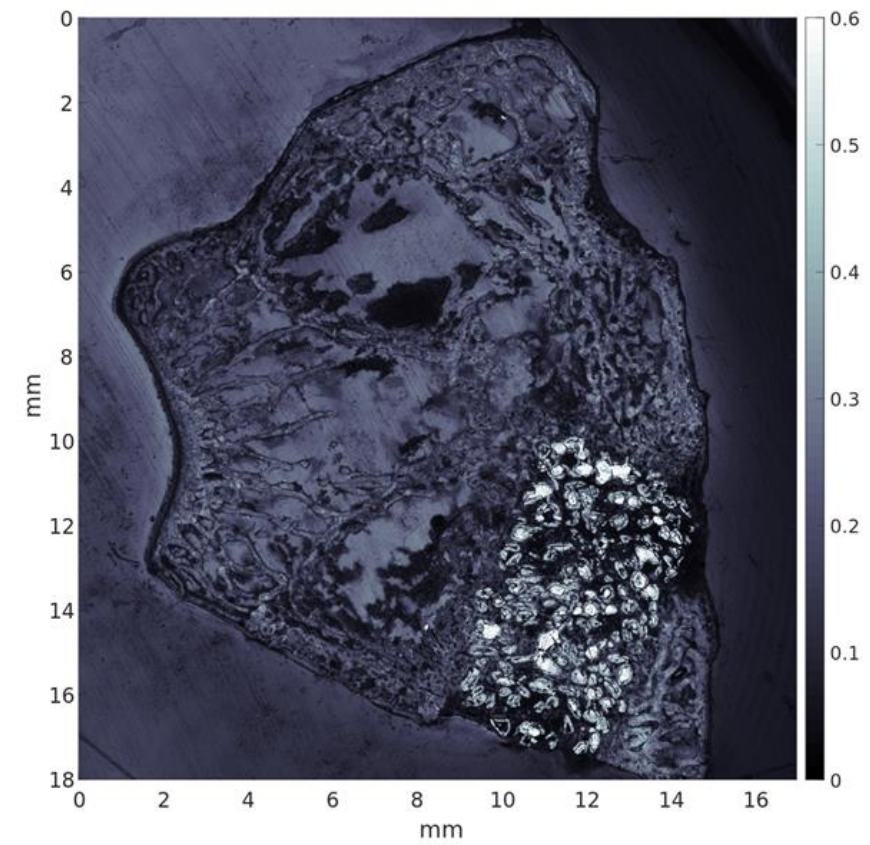

Fig. 2. A CESAM image of an epoxy cast rabbit methaphyseal femur sample showing the implanted BAG-S53P4 in the bottom right. The image has $1800 \mathrm{x}$ 1700 measurement points with $10 \mu \mathrm{m}$ stepping. The color-coding is linear amplitude. This image is constructed from 10 focus layers taken from $10 \mu \mathrm{m}$ apart from each other.

\section{B. Example 2: Non-fixed BAG-S53P4 femur sample}

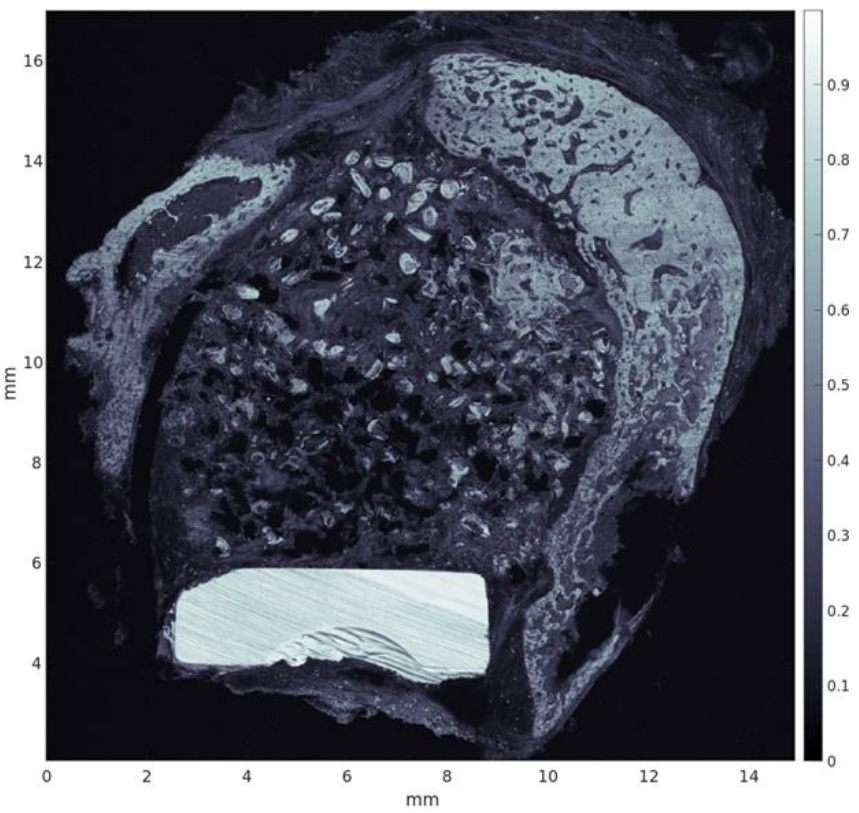

Fig. 3. A CESAM image of a non-fixed diaphyseal femur sample. The image has $1700 \times 1700$ pixels taken with $10 \mu \mathrm{m}$ steps and is constructed from 15 focal layers all $15 \mu \mathrm{m}$ apart from each other. The color coding is the normalized natural logarithm of the focal amplitude.

In Fig. 3, the outer layer of the bone structure resembles denser bone, which is characteristic for callus formation during bone healing at the diaphysis of long bones. The implant with BAG granules is clearly visible, as well as the metal plate. In part of the BAG granules also reaction layers can be observed. Outside the implant surrounding the denser bone tissue, also tissue resembling muscle tissue can be observed.

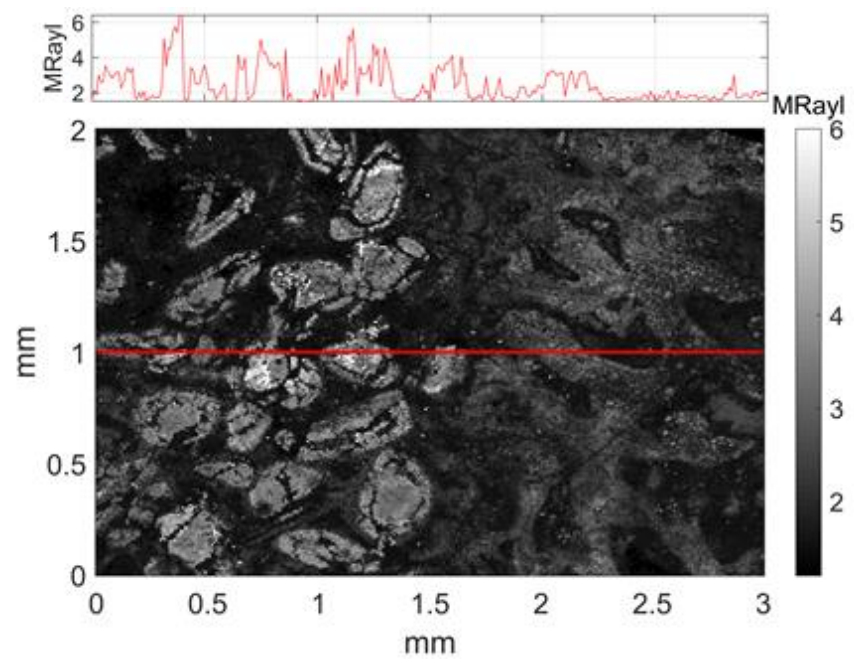

Fig. 4. Acoustic impedance map of a subsection of Fig 2, showing the border between spongy bone and the BAG-S53P4 implant. According to the acoustic impedance grayscale, there also seems to be bone ingrowth from the surrounding bone between the granules in the outer $1 / 3$ and $2 / 3$ part of the implant. The plot above shows the acoustic impedance B-scan marked with the red line

\section{CONCLUSIONS}

We used our custom-made acoustic microscope for large area scanning: we visualized the BAG-S53P4, the bone formation after implantation, as well as the bone structure in two rabbit femur samples. We demonstrated the method for large area scanning with focus layer alignment. We showed that it reduces somewhat the sample flatness requirements. Absolute acoustic impedance values were calculated from the measured data via acoustic impedance calibration. Our device is capable of $250+\mathrm{mm}^{2}$ area imaging with $10 \mu \mathrm{m} \mathrm{XY}$-stepping, with sample surface height variation of over $100 \mu \mathrm{m}$. Large area high resolution scans of biologic samples may find use across a large spectrum of cases, not detailed here.

\section{ACKNOWLEDGEMENTS}

We gratefully acknowledge docent Jukka Pajarinen and graduate students Elin Eriksson, Robert Björkenheim and Gustav Strömberg for their assistance.

\section{REFERENCES}

[1] O'Donnell M. Coded excitation systems for improving the penetration of real-time phased-array imaging systems. IEEE Trans. Ultrason., Ferroelect., Freq. Contr. 1992; vol. 39:341-351.

[2] Björkenheim R, Strömberg G, Pajarinen J, Ainola M, Uppstu P, Hupa L, Bohling TO, Lindfors NC. Polymer-coated bioactive glass S53P4 increases VEGF and TNF expression in an induced membrane model in vivo. Journal of Materials Science 2017;52:9055-9065.

[3] Munukka E, Leppäranta O, Korkeamäki M, Vaahtio M, Peltola T, Zhang D, Hupa L, Ylänen H, Salonen JI, Viljanen MK, Eerola E. Bactericidal effects of bioactive glasses on clinically important aerobic bacteria. $J$ Mater Sci: Mater Med 2008;19:27-32.

[4] Björkenheim R, Strömberg G, Ainola M, Uppstu P,Aalto-Setälä L, Hupa $\mathrm{L}$, Pajarinen J, Lindfors NC. Bone morphogenetic protein expression and bone formation are induced by bioactive glass iv-vivo. J Biomed Mater Res B Appl Biomater 2019;107:847-857. 
[5] A. I. Meriläinen, V. Kananen, C. Fridlund, J. Eskelinen, E. Hæggström and K. Raum, "GHz ultrasonics with arbitrary code excitation," 2013 IEEE International Ultrasonics Symposium (IUS), Prague, 2013, pp. 703706. doi: 10.1109/ULTSYM.2013.0181

[6] K. Raum, K. V. Jenderka, A. Klemenz and J. Brandt, "Multilayer analysis: quantitative scanning acoustic microscopy for tissue characterization at a microscopic scale," in IEEE Transactions on Ultrasonics, Ferroelectrics, and Frequency Control, vol. 50, no. 5, pp. 507-516, May 2003. doi: 10.1109/TUFFC.2003.1201463

[7] NN. Lindfors N.C.\& Aho A.J., Granule size and composition of bioactive glasses affect osteoconduction in rabbit. J. Mater. Sci. Mater. Med. 14, 365-376 (2003). 